Global Journal of Pure and Applied Mathematics.

ISSN 0973-1768 Volume 11, Number 4 (2015), pp. 2381-2392

(C) Research India Publications

https://dx.doi.org/10.37622/GJPAM/11.4.2015.2381-2392

\title{
Direct Theorems for Modified Baskakov Stancu Operators in Simultaneous Approximation
}

\author{
Prerna Maheshwari Sharma and Sangeeta Garg \\ Department of Mathematics, SRM University, NCR Campus; Ghaziabad; India. \\ Mewar University, Chittorgarh (Rajasthan), India
}

\begin{abstract}
In the present paper, we extend our study for modified Baskakov operators defined by Gupta-Srivastava [5]. We introduce modified Baskakov-Stancu type operators and give the moments in terms of hypergeometric series functions. Further, we establish asymptotic formula and error estimation in simultaneous approximation for these operators.
\end{abstract}

Key Words: Hypergeometric series functions, Stancu type generalization, Modified Baskakov operators, Simultaneous approximation, Voronovskaja type asymptotic formula, Schwarz inequality.

AMS Subject Classification: 41A25, 41A35.

\section{Introduction}

In the year 1985, Sahai-Prasad [8] proposed modified Lupas operators for all functions integrable on $[0, \infty)$

$$
Q_{n, m}(x)=(n-1) \sum_{v=0}^{\infty} p_{n, v}(x) \int_{0}^{\infty} p_{n, v}(t) f(t) d t,
$$

where

$p_{n, v}(t)=\left(\begin{array}{c}n+v-1 \\ v\end{array}\right) t^{v}(1+t)^{-(n+v)}=\frac{(n)_{v}}{v !} \frac{x^{v}}{(1+x)^{n+v}}$.

Here $(n)_{v}$ is rising factorial (Pochhammer symbol) and defined as 
$(n)_{v}=n(n+1)(n+2) \ldots(n+v-1)=\frac{(n+v-1) !}{(n-1) !}$.

In 1991, Sinha et al. [9] studied it as modified Baskakov operators. Several researchers- Aniol et al. [1], Gupta et al. [2], [3], [4] estimated the rate of convergence for these operators. In 2009, some approximation properties of modified Baskakov operators were discussed by P. Maheshwari [6]. Recently Maheshwari-Sharma [7] have obtained approximations for $q$-Baskakov Beta Stancu type operators. Srivastava et al. [10], [11] also studied some approximation properties of linear positive operators. Motivating the recent studies, we extend our study and find some direct results on modified Baskakov-Stancu operators using hypergeometric series functions. We write the operator (1.1) as

$$
\begin{aligned}
& Q_{n}(f, x)=(n-1) \sum_{v=0}^{\infty} \frac{(n)_{v}}{v !} \frac{x^{v}}{(1+x)^{n+v}} \int_{0}^{\infty} \frac{(n)_{v}}{v !} \frac{t^{v}}{(1+t)^{n+v}} f(t) d t \\
& =(n-1) \int_{0}^{\infty} \frac{f(t)}{[(1+x)(1+t)]^{n}} \sum_{v=0}^{\infty} \frac{(n)_{v}(n)_{v}}{(v !)^{2}}\left[\frac{x t}{(1+x)(1+t)}\right]^{v} d t,
\end{aligned}
$$

by applying hypergeometric series

$$
{ }_{2} F_{1}(a, b ; c ; x)=\sum_{v=0}^{\infty} \frac{(a)_{v}(b)_{v}}{(c)_{v}} \cdot \frac{x^{v}}{v !} \quad \text { and the identity }(1)_{v}=1 .
$$

We have

$$
Q_{n}(f, x)=(n-1) \int_{0}^{\infty} \frac{f(t)}{[(1+x)(1+t)]^{n}}{ }_{2} F_{1}\left(n, n ; 1 ; \frac{x t}{(1+x)(1+t)}\right) d t .
$$

Now in (1.2), applying Pfaff-Kummer's transformation, defined below

$$
{ }_{2} F_{1}(a, b ; c ; x)=(1-x)^{-a}{ }_{2} F_{1}\left(a, c-b ; c ; \frac{x}{1-x}\right) .
$$

We have

$$
Q_{n}(f, x)=(n-1) \int_{0}^{\infty} \frac{f(t)}{(1+x+t)^{n}}{ }_{2} F_{1}\left(n, 1-n ; 1 ; \frac{-x t}{1+x+t}\right) d t . \ldots
$$

This is the required alternate form of the operators (1.1) in terms of hypergeometric series functions. In 1983, Stancu type generalization [12] of Bernstein operators based on two parameters $\alpha$ and $\beta$ satisfying the condition $0 \leq \alpha \leq \beta$, has been studied by several authors. Motivating the recent work, we propose Stancu type generalization of modified Baskakov operators as

$$
Q_{n}^{\alpha, \beta}(f, x)=(n-1) \int_{0}^{\infty} f\left(\frac{n t+\alpha}{n+\beta}\right){ }_{2} F_{1}\left(n, 1-n ; 1 ; \frac{-x t}{1+x+t}\right) \frac{d t}{(1+x+t)^{n}} .
$$

As a special case, if $\alpha=\beta=0$, modified Baskakov-Stancu operators (1.5) reduce to modified Baskakov operators defined in (1.4). Further we consider 
$C_{\gamma}[0, \infty)=\left\{f \in C[0, \infty): f(t)=O\left(t^{\gamma}\right), \gamma>0\right\}$

so that the operators $Q_{n}^{\alpha, \beta}$ are well defined.

In the present article, we study direct theorems including Voronovskaja type asymptotic formula and an error estimation in simultaneous approximation theory for MBS operators (1.5).

\section{Moment Estimation and Auxiliary Results}

In this section we estimate certain basic results with the use of hypergeometric series functions.

Lemma 1. For $n>0$ and $r>-1$, we have

$$
Q_{n}\left(t^{r}, x\right)=\frac{\Gamma(n-r-1) \Gamma(r+1)}{\Gamma(n-1)}(1+x)^{r}{ }_{2} F_{1}\left(1-n,-r ; 1 ; \frac{x}{1+x}\right) \text {. }
$$

Proof. Substituting $f(t)=t^{r}$ and $t=(1+x) u$ in (1.5),

$$
\begin{aligned}
& Q_{n}\left(t^{r}, x\right)=(n-1) \int_{0}^{\infty} \frac{u^{r}}{(1+u)^{n}}(1+x)^{r-n+1}{ }_{2} F_{1}\left(n, 1-n ; 1 ; \frac{-u x}{1+u}\right) d t \\
& =(n-1) \sum_{v=0}^{\infty} \frac{(n)_{v}(1-n)_{v}}{(v !)^{2}}(-x)^{v}(1+x)^{r-n+1} \int_{0}^{\infty} \frac{u^{r+v}}{(1+u)^{n+v}} d u \\
& =(1+x)^{r-n+1} \sum_{v=0}^{\infty} \frac{\Gamma(n+v)(1-n)_{v}}{\Gamma(n-1)(v !)^{2}}(-x)^{v} \times \frac{\Gamma(v+r+1) \Gamma(n-r-1)}{\Gamma(n+v)}
\end{aligned}
$$

$\operatorname{Using} \Gamma(n+v+1)=\Gamma(n+1)(n+1)_{v}$, we get

$$
\begin{aligned}
& Q_{n}\left(t^{r}, x\right)=(1+x)^{r-n+1} \sum_{v=0}^{\infty} \frac{(1-n)_{v}}{\Gamma(n-1)(v !)^{2}}(-x)^{v} \Gamma(n-r-1) \Gamma(r+1)(r+1)_{v} \\
& =\frac{\Gamma(n-r-1) \Gamma(r+1)}{\Gamma(n-1)}(1+x)^{r-n+1} \sum_{v=0}^{\infty} \frac{(1-n)_{v}(r+1)_{v}}{(v !)^{2}}(-x)^{v} \\
& =\frac{(n-r-2) ! r !}{(n-2) !}(1+x)^{r-n+1}{ }_{2} F_{1}(1-n, 1+r ; 1 ;-x) .
\end{aligned}
$$

Applying Pfaff-Kummer's transformation, we have

$$
\begin{aligned}
& Q_{n}\left(t^{r}, x\right)=\frac{(n-r-2) ! r !}{(n-2) !}(1+x)^{r}{ }_{2} F_{1}\left(1-n,-r ; 1 ; \frac{x}{1+x}\right) \\
& =\frac{\Gamma(n-r-1) \Gamma(r+1)}{\Gamma(n-1)}(1+x)^{r}{ }_{2} F_{1}\left(1-n,-r ; 1 ; \frac{x}{1+x}\right) .
\end{aligned}
$$

Lemma 2. For $0 \leq \alpha \leq \beta$, we have 
$Q_{n}^{\alpha, \beta}(f, x)=x^{r} \frac{n^{r}}{(n+\beta)^{r}} \frac{(n+r-1) !(n-r-2) !}{(n-1) !(n-2) !}+r \frac{n^{r}}{(n+\beta)^{r}}\left[r \frac{(n+r-2) !(n-r-2) !}{(n-1) !(n-2) !}\right.$

$\left.+\frac{\alpha}{n} \frac{(n+r-2) !(n-r-1) !}{(n-1) !(n-2) !}\right] x^{r-1}+\frac{n^{r-1}}{(n+\beta)^{r}} \frac{r(r-1)}{2} \alpha^{2} \times$

$\left[\frac{(r-1)}{\alpha} \frac{(n+r-3) !(n-r-1) !}{(n-1) !(n-2) !}+\frac{1}{n} \frac{(n+r-3) !(n-r) !}{(n-1) !(n-2) !}\right]+O\left(n^{-2}\right)$.

Proof. From the relation (1.5)

$Q_{n}^{\alpha, \beta}(f, x)=(n-1) \int_{0}^{\infty}\left(\frac{n t+\alpha}{n+\beta}\right)^{r}{ }_{2} F_{1}\left(n, 1-n ; 1 ; \frac{-x t}{1+x+t}\right) \frac{d t}{(1+x+t)^{n}}$

$=\sum_{j=0}^{r}\left(\begin{array}{l}r \\ j\end{array}\right) \frac{n^{j} \alpha^{r-j}}{(n+\beta)^{r}}(n-1) \int_{0}^{\infty} \frac{t^{j}}{(1+x+t)^{n}}{ }_{2} F_{1}\left(n, 1-n ; 1 ; \frac{-x t}{1+x+t}\right) d t$

$=\sum_{j=0}^{r}\left(\begin{array}{l}r \\ j\end{array}\right) \frac{n^{j} \alpha^{r-j}}{(n+\beta)^{r}} Q_{n}\left(t^{j}, x\right)$,

$(\operatorname{from}(1.2))$

$=\frac{1}{(n+\beta)^{r}}\left[n^{r} Q_{n}\left(t^{r}, x\right)+r n^{r-1} \alpha Q_{n}\left(t^{r-1}, x\right)+\frac{r(r-1)}{2} n^{r-2} \alpha^{2} Q_{n}\left(t^{r-2}, x\right)\right]+O\left(n^{-2}\right)$.

Using Lemma 1, we have

$Q_{n}^{\alpha, \beta}(f, x)=\frac{1}{(n+\beta)^{r}}\left[n^{r}\left\{\frac{(n-r-2) !(n+r-1) !}{(n-1) !(n-2) !} x^{r}+\frac{(n-r-2) !(n+r-1) !}{(n-1) !(n-2) !} r^{2} x^{r-1}\right\}\right.$

$+\alpha n^{r-1}\left\{\frac{(n-r-1) !(n+r-2) !}{(n-1) !(n-2) !} r x^{r-1}+(r-1)^{2} \frac{(n-r-1) !(n+r-3) !}{(n-1) !(n-2) !} x^{r-2}\right\}$

$\left.+\frac{r(r-1)}{2} n^{r-2} \alpha^{2} \frac{(n-r) !(n+r-3) !}{(n-1) !(n-2) !} x^{r-2}\right]+O\left(n^{-2}\right)$.

Collecting the coefficients of $x^{r}, x^{r-1}$ and $x^{r-2}$, we obtain the required result.

Lemma 3. [5] For $m \in N \cup\{0\}$, if

$U_{n, m}(x)=\sum_{v=0}^{\infty} p_{n, v}(x)\left(\frac{v}{n}-x\right)^{m}$,

then $U_{n, 0}(x)=1$ and $U_{n, 1}(x)=0$ and the recurrence formula

$n U_{n, m+1}(x)=x(1+x)\left[U_{n, m}^{\prime}(x)+m U_{n, m-1}(x)\right]$

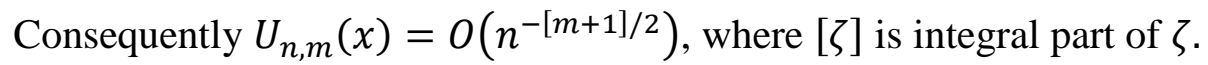

Lemma 4. We define the central moments as 


$$
M_{n, m}^{\alpha, \beta}(f, x)=Q_{n}^{\alpha, \beta}\left((t-x)^{m}, x\right)=(n-1) \sum_{v=0}^{\infty} p_{n, v}(x) \int_{0}^{\infty} p_{n, v}(t)\left(\frac{n t+\alpha}{n+\beta}-x\right)^{m} d t,
$$

then we have $M_{n, 0}^{\alpha, \beta}(f, x)=1$ and $M_{n, 1}^{\alpha, \beta}(f, x)=\frac{n(1-x+n x)-(n-2)(\alpha-\beta x)}{(n-2)(n+\beta)}$,

for all $n \in N$. Moreover the following recurrence relation holds

$$
\begin{aligned}
& (n-m-2)\left(\frac{n+\beta}{n}\right) M_{n, m+1}^{\alpha, \beta}(x) \\
& =x(1+x)\left[M_{n, m}^{\prime \alpha, \beta}(x)+m M_{n, m-1}^{\alpha, \beta}(x)\right]+\left(\frac{\alpha}{n+\beta}-x\right)\left[\left(\frac{n+\beta}{n}\right)\left(\frac{\alpha}{n+\beta}-x\right)-1\right] M_{n, m-1}^{\alpha, \beta}(x) \\
& +\left[(m+n x+1)+\left(\frac{n+\beta}{n}\right)\left(\frac{\alpha}{n+\beta}-x\right)(n-2 m-2)\right] M_{n, m}^{\alpha, \beta}(x)
\end{aligned}
$$

and $M_{n, m}(x)=O\left(n^{-[m+1] / 2}\right)$ for all $x \in[0, \infty)$, where $[\alpha]$ is integral part of $\alpha$.

Proof. By the definition of the operators (1.5), we get $M_{n, 0}^{\alpha, \beta}(x)=1$. Other moments can also be obtained easily. For the recurrence relation, we follow as

$$
M_{n, m}^{\prime, \alpha, \beta}(x)+m M_{n, m-1}^{\alpha, \beta}(x)=(n-1) \sum_{v=0}^{\infty} p_{n, v}^{\prime}(x) \int_{0}^{\infty} p_{n, v}(t)\left(\frac{n t+\alpha}{n+\beta}-x\right)^{m} d t .
$$

Using $x(1+x) p_{n, v}^{\prime}(x)=(v-n x) p_{n, v}(x)$, we get

$$
\begin{aligned}
& x(1+x)\left[M_{n, m}^{\prime \alpha, \beta}(x)+m M_{n, m-1}^{\alpha, \beta}(x)\right] \\
& =(n-1) \sum_{v=0}^{\infty}(v-n x) p_{n, v}(x) \int_{0}^{\infty} p_{n, v}(t)\left(\frac{n t+\alpha}{n+\beta}-x\right)^{m} d t \\
& =(n-1) \sum_{v=0}^{\infty}(v-n x) p_{n, v}(x) \int_{0}^{\infty}[(v-n t)+n t-n x] p_{n, v}(t)\left(\frac{n t+\alpha}{n+\beta}-x\right)^{m} d t \\
& =(n-1) \sum_{v=0}^{\infty} p_{n, v}(x) \int_{0}^{\infty} t(1+t) p_{n, v}(t)\left(\frac{n t+\alpha}{n+\beta}-x\right)^{m} d t+n(n-1) \\
& \times \sum_{v=0}^{\infty}(v-n x) p_{n, v}(x) \int_{0}^{\infty} t p_{n, v}(t)\left(\frac{n t+\alpha}{n+\beta}-x\right)^{m} d t-n x M_{n, m}^{\alpha, \beta}(x) .
\end{aligned}
$$

Substituting $t=\frac{n+\beta}{n}\left[\left(\frac{n t+\alpha}{n+\beta}-x\right)-\left(\frac{\alpha}{n+\beta}-x\right)\right]$, we have

$$
\begin{aligned}
& x(1+x)\left[M_{n, m}^{\prime, \alpha, \beta}(x)+m M_{n, m-1}^{\alpha, \beta}(x)\right] \\
& =\left(\frac{n+\beta}{n}\right)\left[(n-1) \sum_{v=0}^{\infty} p_{n, v}(x) \int_{0}^{\infty} p_{n, v}^{\prime}(t)\left(\frac{n t+\alpha}{n+\beta}-x\right)^{m+1} d t-\left(\frac{\alpha}{n+\beta}-x\right)(n-1)\right.
\end{aligned}
$$




$$
\begin{aligned}
& \left.\times \sum_{v=0}^{\infty} p_{n, v}(x) \int_{0}^{\infty} p_{n, v}^{\prime}(t)\left(\frac{n t+\alpha}{n+\beta}-x\right)^{m} d t\right]+\left(\frac{n+\beta}{n}\right)^{2}\left[(n-1) \sum_{v=0}^{\infty} p_{n, v}(x) \int_{0}^{\infty} p_{n, v}^{\prime}(t)\right. \\
& \times\left(\frac{n t+\alpha}{n+\beta}-x\right)^{m+2} d t+\left(\frac{\alpha}{n+\beta}-x\right)^{2}(n-1) \sum_{v=0}^{\infty} p_{n, v}(x) \int_{0}^{\infty} p_{n, v}^{\prime}(t)\left(\frac{n t+\alpha}{n+\beta}-x\right)^{m} d t \\
& \left.-2\left(\frac{\alpha}{n+\beta}-x\right)(n-1) \sum_{v=0}^{\infty} p_{n, v}(x) \int_{0}^{\infty} p_{n, v}^{\prime}(t)\left(\frac{n t+\alpha}{n+\beta}-x\right)^{m+1} d t\right]+(n+\beta) \\
& \times\left[M_{n, m+1}^{\alpha, \beta}(x)-\left(\frac{\alpha}{n+\beta}-x\right) M_{n, m}^{\alpha, \beta}(x)\right]-n x M_{n, m}^{\alpha, \beta}(x) \\
& =\left(\frac{n+\beta}{n}\right)\left[-(m+1) M_{n, m}^{\alpha, \beta}(x)+\left(\frac{\alpha}{n+\beta}-x\right) m M_{n, m-1}^{\alpha, \beta}(x)\right]+\left(\frac{n+\beta}{n}\right)^{2}[-(m+2) \\
& \left.\times M_{n, m+1}^{\alpha, \beta}(x)-m\left(\frac{\alpha}{n+\beta}-x\right)^{2} M_{n, m-1}^{\alpha, \beta}(x)+2\left(\frac{\alpha}{n+\beta}-x\right)(m+1) M_{n, m}^{\alpha, \beta}(x)\right] \\
& +(n+\beta)\left[M_{n, m+1}^{\alpha, \beta}(x)-\left(\frac{\alpha}{n+\beta}-x\right) M_{n, m}^{\alpha, \beta}(x)\right]-n x M_{n, m}^{\alpha, \beta}(x) .
\end{aligned}
$$

On arranging the like terms, we get the desired recurrence formula.

Lemma 5. [5] There exists the polynomial $q_{i, j, r}(x)$ on [0, $\left.\infty\right)$, independent of $n$ and $v$ such that

$$
x^{r}(1+x)^{r} \frac{d^{r}}{d x^{r}} p_{n, v}(x)=\sum_{\substack{2 i+j \leq r, i, j \geq 0}} n^{i}(v-n x)^{j} q_{i, j, r}(x) p_{n, v}(x) .
$$

\section{Direct Estimates}

In this section, we present some important estimation theorems in simultaneous approximation.

Theorem1. Let $\mathrm{f} \in \mathrm{C}_{\gamma}[0, \infty)$ be bounded on every finite subinterval of $[0, \infty)$, taking the derivative of order $(r+2)$ at fixed $x \in[0, \infty)$. Consider $f(t)=O\left(t^{\gamma}\right)$ as $t \rightarrow$ $\infty$, for some $\gamma>0$, we have

$$
\begin{aligned}
& \lim _{n \rightarrow \infty} n\left[Q_{n, \alpha, \beta}^{(r)}(f, x)-f^{(r)}(x)\right]=r(1+r-\beta) f^{(r)}(x)+[(1+r+\alpha)+x(2+2 r-\beta)] \\
& \times f^{(r+1)}(x)+x(1+x) f^{(r+2)}(x) .
\end{aligned}
$$

Proof. Taylor expansion of $f$ is given as

$$
f(t)=\sum_{i=0}^{r+2} \frac{f^{(i)}(x)}{i !}(t-x)^{i}+\epsilon(t, x)(t-x)^{r+2},
$$


where $\epsilon(t, x) \rightarrow 0$ and $\epsilon(t, x) \rightarrow O\left((t-x)^{\delta}\right)$ as $t \rightarrow \infty$ for some $\delta>0$. Hence $n\left[Q_{n, \alpha, \beta}^{(r)}(f, x)-f^{(r)}(x)\right]$

$=n\left[\left\{\sum_{i=0}^{r+2} \frac{f^{(i)}(x)}{i !} Q_{n, \alpha, \beta}^{(r)}\left((t-x)^{i}, x\right)-f^{(r)}(x)\right\}+Q_{n, \alpha, \beta}^{(r)}\left(\epsilon(t, x)(t-x)^{r+2}, x\right)\right]$

$=J_{1}+J_{2}$.

From Lemma 2, we get

$$
\begin{aligned}
& J_{1}=n \sum_{i=0}^{r+2} \frac{f^{(i)}(x)}{i !} \sum_{j=r}^{i}\left(\begin{array}{l}
i \\
j
\end{array}\right)(-x)^{i-j} Q_{n, \alpha, \beta}^{(r)}\left(t^{j}, x\right)-n f^{(r)}(x) \\
& =n \frac{f^{(r)}(x)}{r !}\left[Q_{n, \alpha, \beta}^{(r)}\left(t^{r}, x\right)-r !\right]+n \frac{f^{(r+1)}(x)}{(r+1) !}\left[(r+1)(-x) Q_{n, \alpha, \beta}^{(r)}\left(t^{r}, x\right)+Q_{n, \alpha, \beta}^{(r)}\left(t^{r+1}, x\right)\right] \\
& +n \frac{f^{(r+2)}(x)}{(r+2) !}\left[\frac{(r+1)(r+2)}{2 !} x^{2} Q_{n, \alpha, \beta}^{(r)}\left(t^{r}, x\right)-(r+2) x Q_{n, \alpha, \beta}^{(r)}\left(t^{r+1}, x\right)+Q_{n, \alpha, \beta}^{(r)}\left(t^{r+2}, x\right)\right] \\
& =n f^{(r)}(x)\left[\frac{n^{r}}{(n+\beta)^{r}} \frac{(n+r-1) !(n-r-2) !}{(n-1) !(n-2) !}-1\right]+\frac{n f^{(r+1)}(x)}{(r+1) !} \times \frac{n^{r}}{(n+\beta)^{r}}[(r+1)(-x) \\
& \times \frac{(n+r-1) !(n-r-2) !}{(n-1) !(n-2) !} r !+\frac{n}{n+\beta}\left(\frac{(n+r) !(n-r-3) !}{(n-1) !(n-2) !}(r+1) ! x+(r+1)^{2}\right. \\
& \left.\left.\times \frac{(n+r-1) !(n-r-3) !}{(n-1) !(n-2) !} r !\right)+\frac{(r+1) \alpha}{n+\beta} \frac{(n+r-1) !(n-r-2) !}{(n-1) !(n-2) !} r !\right]+\frac{n f^{(r+2)}(x)}{(r+2) !} \\
& \times \frac{n^{r}}{(n+\beta)^{r}}\left[\frac{(r+1)(r+2)}{2 !} \frac{(n+r-1) !(n-r-2) !}{(n-1) !(n-2) !} r ! x^{2}-(r+2) x\left\{\frac{n}{n+\beta}\right.\right. \\
& \times\left(\frac{(n+r) !(n-r-3) !}{(n-1) !(n-2) !}(r+1) ! x+\frac{(n+r-1) !(n-r-3) !}{(n-1) !(n-2) !} r !\right)+\frac{(r+1) \alpha}{n+\beta} \\
& \left.\times \frac{(n+r-1) !(n-r-2) !}{(n-1) !(n-2) !} r !\right\}+\left(\frac{n}{n+\beta}\right)^{2}\left\{\frac{(n+r+1) !(n-r-4) !}{(n-1) !(n-2) !} \frac{(r+2) !}{2} x^{2}+(r+2)^{2}\right. \\
& \left.\times \frac{(n+r) !(n-r-2) !}{(n-1) !(n-2) !}(r+1) ! x\right\}+(r+2) \alpha \frac{n}{(n+\beta)^{2}}\left\{\frac{(n+r) !(n-r-3) !}{(n-1) !(n-2) !}(r+1) ! x+\right. \\
& \left.\left.(r+1)^{2} \frac{(n+r-1) !(n-r-3) !}{(n-1) !(n-2) !} r !\right\}+\frac{(r+1)(r+2)}{2 !} \frac{\alpha^{2}}{(n+\beta)^{2}} \frac{(n+r-1) !(n-r-2) !}{(n-1) !(n-2) !} r !\right] \\
& +O\left(n^{-2}\right) \text {. }
\end{aligned}
$$

Substituting $r=1,2, \ldots$ in the coefficients of $f^{(r)}$ and then taking limit as $n \rightarrow \infty$, we get the coefficient of $f^{(1)}(x)=(2-\beta)=1(1+1-\beta)$,

the coefficient of $f^{(2)}(x)=(6-2 \beta)=2(1+2-\beta)$ and so on.

Thus by induction, the coefficients of $f^{(r)}(x), f^{(r+1)}(x)$ and $f^{(r+2)}(x)$ are $r(1+$ $r-\beta), \quad(1+r+\alpha)+x(2+2 r-\beta)$ and $x(1+x)$ respectively. In order to complete the proof, we have to show that $J_{2} \rightarrow 0$ as $n \rightarrow \infty$. Therefore using Lemma 5 , 
$\left|J_{2}\right| \leq n^{i+1}(n-1) \sum_{\substack{2 i+j \leq r, i, j \geq 0}} \frac{\left|q_{i, j, r}(x)\right|}{x^{r}(1+x)^{r}} \sum_{v=0}^{\infty} p_{n, v}(x)|v-n x|^{j}$

$\times \sum_{v=0}^{\infty} p_{n, v}(x) \int_{0}^{\infty} p_{n, v}(t)|\epsilon(t, x)|\left|\frac{n t+\alpha}{n+\beta}-x\right|^{r+2} d t$.

Since $\epsilon(t, x) \rightarrow 0$ as $t \rightarrow x$ for a given $\epsilon>0$, there exists $\delta>0$ such that $|\epsilon(t, x)|<\epsilon$ whenever $|t-x|<\delta$. Further, if $\lambda>\max \{\gamma, r+2\}$, where $\lambda$ is any integer. Then for all $|t-x| \geq \delta$, we can find a constant $M>0$ such that $|\epsilon(t, x)|\left|\frac{n t+\alpha}{n+\beta}-x\right|^{r+2} \leq M\left|\frac{n t+\alpha}{n+\beta}-x\right|^{\lambda}$.

Therefore from above, we have

$\left|J_{2}\right| \leq C_{1} \sum_{\substack{2 i+j \leq r, i, j \geq 0}} n^{i+1}(n-1) \sum_{v=0}^{\infty} p_{n, v}(x)|v-n x|^{j} \times$

$\left\{\int_{|t-x|<\delta} \epsilon p_{n, v}(t)\left|\frac{n t+\alpha}{n+\beta}-x\right|^{r+2} d t+\int_{|t-x| \geq \delta} M p_{n, v}(t)\left|\frac{n t+\alpha}{n+\beta}-x\right|^{r+2} d t\right\}$ $=J_{3}+J_{4}$.

Applying Schwarz inequality for integration and summation, we get

$$
\begin{aligned}
& \left|J_{3}\right| \leq \epsilon C_{1} \sum_{\substack{2 i+j \leq r, i, j \geq 0}} n^{i+1} \sum_{v=0}^{\infty} p_{n, v}(x)|v-n x|^{j}\left\{(n-1) \int_{0}^{\infty} p_{n, v}(t) d t\right\}^{1 / 2} \\
& \times\left\{(n-1) \int_{0}^{\infty} p_{n, v}(t)\left|\frac{n t+\alpha}{n+\beta}-x\right|^{2 r+4} d t\right\}^{1 / 2} \\
& \leq \epsilon C_{1} \sum_{2 i+j \leq r,} n^{i+1}\left\{\sum_{v=0}^{\infty}(n-1) p_{n, v}(x)|v-n x|^{2 j}\right\}^{1 / 2} \\
& \quad \times\left\{(n-1) \sum_{v=0}^{\infty} p_{n, v}(x) \int_{0}^{\infty} p_{n, v}(t)\left|\frac{n t+\alpha}{n+\beta}-x\right|^{2 r+4} d t\right\}^{1 / 2} .
\end{aligned}
$$

For arbitrary $\epsilon$, by using Lemma 3 and Lemma 4, we get $\left\|J_{3}\right\| \leq \epsilon C_{1} \sum_{\substack{2 i+j \leq r, i, j \geq 0}} n^{i+1} O\left(n^{j / 2}\right) O\left(n^{-[r+2] / 2}\right)=\epsilon O(1)=o(1)$.

Applying Schwarz inequality in $J_{4}$, 


$$
\begin{aligned}
& \left|J_{4}\right| \leq C_{2} \sum_{\substack{2 i+j \leq r, i, j \geq 0}} n^{i+1}(n-1) \sum_{v=0}^{\infty} p_{n, v}(x)|v-n x|^{j} \int_{0}^{\infty} p_{n, v}(t)\left|\frac{n t+\alpha}{n+\beta}-x\right|^{\lambda} d t \\
& \leq C_{2} \sum_{\substack{2 i+j \leq r, i, j \geq 0}} n^{i+1}\left\{(n-1) \sum_{v=0}^{\infty} p_{n, v}(x)|v-n x|^{2 j}\right\}^{1 / 3} \\
& \times\left\{(n-1) \sum_{v=0}^{\infty} p_{n, v}(x) \int_{0}^{\infty} p_{n, v}(t)\left|\frac{n t+\alpha}{n+\beta}-x\right|^{2 \lambda} d t\right\}^{1 / 2} .
\end{aligned}
$$

.Using Lemma 3 and Lemma 4, we obtain

$\left\|J_{4}\right\| \leq C_{2} \sum_{\substack{2 i+j \leq r, i, j \geq 0}} n^{i+1} O\left(n^{j / 2}\right) O\left(n^{-\lambda / 2}\right)=C_{2} O\left(n^{(r+2-\lambda) / 2}\right)=o(1)$,

where $C_{1}$ and $C_{2}$ are arbitrary constants. Now $J_{2} \rightarrow 0$ as $n \rightarrow \infty$. Again combining the estimates $J_{1}$ and $J_{2}$, we get the desired result. Hence the theorem is completed.

Theorem 2. Let $\mathrm{f} \in \mathrm{C}_{\gamma}[0, \infty)$ for some $\gamma>0$ and $r \leq m \leq r+2$. If $f^{(m)}$ exists and is continuous on $(a-\eta, b+\eta) \subset(0, \infty), \eta>0$, then for $n$ sufficiently large, we have

$\left\|Q_{n, \alpha, \beta}^{(r)}(f, x)-f^{(r)}(x)\right\|_{C[a, b]} \leq K_{1} n^{-1} \sum_{i=r}^{m}\left\|f^{(i)}(x)\right\|_{C[a, b]}+K_{2} n^{-1 / 2} \omega\left(f^{(m)}, n^{-1 / 2}\right)+O\left(n^{-2}\right)$

where $K_{1}$ and $K_{2}$ are constants independent of $f$ and $n, \omega(f, \delta)$ is the modulus of continuity of $f$ on $(a-\eta, b+\eta)$ and $\|.\|_{C[a ; b]}$ denotes the sup-norm on $[a, b]$.

Proof. Taylor expansion of $f$ is given by

$f(t)=\sum_{i=0}^{m} \frac{f^{(i)}(x)}{i !}(t-x)^{i}+\frac{f^{(m)}(\xi)-f^{(m)}(x)}{m !}(t-x)^{m} \chi(t)+h(t, x)(1-\chi(t))$,

where $\xi$ is the characteristic function on $(a-\eta, b+\eta)$ and lies between $t$ and $x$. .Therefore

$$
\begin{aligned}
& \left\{Q_{n, \alpha, \beta}^{(r)}(f, x)-f^{(r)}(x)\right\} \\
& =\sum_{i=0}^{m} \frac{f^{(i)}(x)}{i !} Q_{n, \alpha, \beta}^{(r)}\left((t-x)^{i}, x\right)+Q_{n, \alpha, \beta}^{(r)}\left(\frac{f^{(m)}(\xi)-f^{(m)}(x)}{m !}(t-x)^{m} \chi(t), x\right) \\
& +Q_{n, \alpha, \beta}^{(r)}(h(t, x)(1-\chi(t)), x)=E_{1}+E_{2}+E_{3} .
\end{aligned}
$$

Applying Lemma 2, 
$E_{1}=\sum_{i=0}^{m} \frac{f^{(i)}(x)}{i !} \sum_{j=0}^{i}(-x)^{i-j} \frac{d^{r}}{d x^{r}}\left[j \frac{n^{j}}{(n+\beta)^{j}}\left\{j \frac{(n+j-1) !(n-j-2) !}{(n-1) !(n-2) !}+\frac{\alpha}{n}\right.\right.$

$\left.\times \frac{(n+j-2) !(n-j-1) !}{(n-1) !(n-2) !}\right\} x^{j-1}+\frac{j(j-1)^{2}}{2} \alpha \frac{n^{j-1}}{(n+\beta)^{j}}\left\{2 \frac{(n+j-3) !(n-j-1) !}{(n-1) !(n-2) !}\right.$

$\left.\left.+\frac{\alpha}{n(j-1)} \frac{(n+j-3) !(n-j) !}{(n-1) !(n-2) !}\right\} x^{J-2}+O\left(n^{-2}\right)\right]-f^{(r)}(x)$.

Consequently, we have

$\left\|E_{1}\right\|_{C[a, b]} \leq K_{1} n^{-1} \sum_{i=r}^{m}\left\|f^{(i)}(x)\right\|_{C[a, b]}+O\left(n^{-2}\right)$

uniformly on $[a, b]$. To estimate $E_{2}$, we take

$\left|E_{2}\right| \leq(n-1) \sum_{v=0}^{\infty}\left|p_{n, v}^{(r)}(t)\right| \int_{0}^{\infty} p_{n, v}(t)\left|\frac{f^{(m)}(\xi)-f^{(m)}(x)}{m !}\right|\left|\frac{n t+\alpha}{n+\beta}-x\right|^{m} \chi(t) d t$

By the continuity of $f$, we have $\left|f^{(m)}(\xi)-f^{(m)}(x)\right| \leq \omega\left(f^{(m)}, \delta\right)$. Thus

$\left|E_{2}\right| \leq \frac{\omega\left(f^{(m)}, \delta\right)}{\mathrm{m} !}(n-1) \sum_{v=0}^{\infty}\left|p_{n, v}^{(r)}(t)\right| \int_{0}^{\infty} p_{n, v}(t)\left|1-\frac{\frac{n t+\alpha}{n+\beta}-x}{\delta}\right|\left|\frac{n t+\alpha}{n+\beta}-x\right|^{m} d t$

$\leq \frac{\omega\left(f^{(m)}, \delta\right)}{\mathrm{m} !}\left\{(n-1) \sum_{v=0}^{\infty}\left|p_{n, v}^{(r)}(t)\right| \int_{0}^{\infty} p_{n, v}(t)\left|\frac{n t+\alpha}{n+\beta}-x\right|^{m} d t\right.$

$+(n-1) \sum_{v=0}^{\infty}\left|p_{n, v}^{(r)}(t)\right| \int_{0}^{\infty} p_{n, v}(t) \delta^{-1}\left|\frac{n t+\alpha}{n+\beta}-x\right|^{m+1} d t$.

By using Schwarz inequality,

$$
\begin{aligned}
& (n-1) \sum_{v=0}^{\infty}\left|p_{n, v}^{(r)}(t)\right||v-n x|^{j} \int_{0}^{\infty} p_{n, v}(t)\left|\frac{n t+\alpha}{n+\beta}-x\right|^{m} d t \\
& \leq\left\{\sum_{v=0}^{\infty}\left|p_{n, v}^{(r)}(t)\right||v-n x|^{2 j}\right\}^{\frac{1}{2}}\left\{(n-1) \int_{0}^{\infty} p_{n, v}(t) d t\right\}^{\frac{1}{2}}\left\{(n-1) \int_{0}^{\infty} p_{n, v}(t)\left|\frac{n t+\alpha}{n+\beta}-x\right|^{2 m} d t\right\}^{1 / 2} \\
& \leq \mathrm{O}\left(\mathrm{n}^{\mathrm{j} / 2}\right) \cdot \mathrm{O}\left(\mathrm{n}^{-\mathrm{m} / 2}\right)=\mathrm{O}\left(\mathrm{n}^{(\mathrm{j}-\mathrm{m}) / 2}\right) .
\end{aligned}
$$

Therefore, using Lemma 5

$$
(n-1) \sum_{v=0}^{\infty}\left|p_{n, v}^{(r)}(t)\right| \int_{0}^{\infty} p_{n, v}(t)\left|\frac{n t+\alpha}{n+\beta}-x\right|^{m} d t
$$




$$
\begin{aligned}
& \leq \sum_{v=0}^{\infty} \sum_{\substack{2 i+j \leq r, i, j \geq 0}} n^{i}|v-n x|^{j} \frac{\left|q_{i, j, r}(x)\right|}{x^{r}(1+x)^{r}}(n-1) p_{n, v}(x) \int_{0}^{\infty} p_{n, v}(t)\left|\frac{n t+\alpha}{n+\beta}-x\right|^{m} d t \\
& \leq \sup _{\substack{2 i+j \geq r, i, j \geq 0}} \frac{\left|q_{i, j, r}(x)\right|}{x^{r}(1+x)^{r}} \sum_{\substack{2 i+j \leq r, i, j \geq 0}} n^{i}(n-1) \sum_{v=0}^{\infty} p_{n, v}(x)|v-n x|^{j} \int_{0}^{\infty} p_{n, v}(t)\left|\frac{n t+\alpha}{n+\beta}-x\right|^{m} d t \\
& =C \sum_{\substack{2 i+j \leq r, i, j \geq 0}} n^{i} O\left(n^{(j-m) / 2}\right)=O\left(n^{(r-m) / 2}\right)
\end{aligned}
$$

uniformly on $[a, b]$, where

$$
C=\sup _{\substack{2 i+j \geq r, i, j \geq 0}} \sup _{x \in[a, b]} \frac{\left|q_{i, j, r}(x)\right|}{x^{r}(1+x)^{r}}
$$

Taking $\delta=n^{-1 / 2}$ and using (3.1), we get

$\left\|E_{2}\right\|_{C[a, b]} \leq \frac{\omega\left(f^{(m)}, n^{-1 / 2}\right)}{\mathrm{m} !}\left[O\left(n^{(r-m) / 2}\right)+n^{-1 / 2} O\left(n^{(r-m-1) / 2}\right)+O\left(n^{-m}\right)\right]$

$\leq K_{2} O\left(n^{-(r-m) / 2}\right) \omega\left(f^{(m)}, n^{-1 / 2}\right)$.

To estimate $E_{3}$, using Lemma 5 , we have

$$
\left|E_{3}\right| \leq C \sum_{\substack{2 i+j \leq r, i, j \geq 0}} n^{i} \sum_{v=0}^{\infty} p_{n, v}(x)|v-n x|^{j} \int_{0}^{\infty} p_{n, v}(t)|h(t, x)| d t .
$$

Since $t \in[0, \infty) \backslash(a-\eta, b+\eta)$, we can choose $\delta$ such that $|t-x| \geq \delta$, for all $[a, b]$. Now choosing a constant $K$ such that $|h(t, x)| \leq K\left|\frac{n t+\alpha}{n+\beta}-x\right|^{\mu}$, where $\mu \geq$ $\max \{r, m\}$ is an integer. Hence using (3.1), we get $\left\|E_{3}\right\|=O\left(n^{-s}\right)$ for all $s>0$ and uniformly on $[a, b]$.

Combining estimates $E_{1}, E_{2}$ and $E_{3}$, we get the required result.

\section{References}

[1] G. Aniol and P.P. Taberska, "On the rate of pointwise convergence of Durrmeyer type operators", Approx.Theory and its Applications 11(1995), 94105.

[2] V. Gupta and P. Gupta, "Rate of convergence for the Baskakov Durrmeyer operators", Ganita 52(1) (2001), 69-77.

[3] V. Gupta and D. Kumar, "Rate of convergence of modified Baskakov operators", Demonstratio Math. XXX 2(1997), 339-346. 
[4] V. Gupta and P. Maheshwari, "On Baskakov Szasz type operators", Kyungpook Math. J. 43(2003), 315-325..

[5] V. Gupta and G.S. Srivastava, "An estimate on the rate of convergence of modified Baskakov operators for functions of bounded variation”, Kyungpook Math. J. 36 (1996), 237-247.

[6] P. Maheshwari, "Direct theorems for modified Baskakov operators in Lpspace", Demonstratio Math. XLL 3(2009), 533-565.

[7] P. Maheshwari and D. Sharma, "Approximation by $q$-Baskakov-Beta-Stancu operators", Rend. Circ. Mat. Palermo (2012), 61:297-305.

[8] A. Sahai and G. Prasad, "On simultaneous approximation by the modified Lupas operators”, J. Approx.Theory 45(1985), 122-128.

[9] R.P. Sinha, P.N. Agrawal and V. Gupta, "On simultaneous approximation by modified Baskakov operators", Bull. Soc. Math. Belg. Ser. B 42(2) (1991), 217-231.

[10] H.M. Srivastava and V. Gupta, "A certain family of summation integral type operators", Math. Comput. Modelling 37(2003), 1307-1315.

[11] H.M. Srivastava and V. Gupta, "Rate of convergence for the Bezier variant of the Bleimann-Butzer-Hahn operators", Applied Math. Letters 18(2005), 849857.

[12] D.D. Stancu, "Approximation of functions by means of new generalized Bernstein operators", Calcolo 20(1983), 211-229. 